\title{
JULGAMENTO PERCEPTIVO DA GRAVIDADE DO DESVIO FONOLÓGICO POR TRÊS GRUPOS DISTINTOS
}

\author{
Perceptual judgment of severity of phonological disorder assessed by \\ three distinct groups
}

\author{
Gabriele Donicht ${ }^{(1)}$, Karina Carlesso Pagliarin (2), Márcia Keske-Soares ${ }^{(3)}$, Helena Bolli Mota ${ }^{(4)}$
}

\begin{abstract}
RESUMO
Objetivo: comparar a gravidade do desvio fonológico a partir da análise perceptiva de três grupos distintos de julgadoras. Métodos: a pesquisa foi composta de duas amostras, uma julgada, composta por 30 sujeitos com desvio fonológico com idades entre 4:1 e 7:11, e outra julgadora (cinco fonoaudiólogas, cinco leigas e cinco mães). Foi avaliada a fala espontânea das crianças através da narração de três sequências lógicas, as quais foram analisadas pelas julgadoras, acompanhadas por uma grade para marcação da gravidade. Para análise dos dados, foi realizada a Moda das 90 narrativas, possibilitando a análise estatística dos dados através da Análise de Concordância-Kappa. Determinou-se a gravidade do desvio fonológico pelo Percentual de Consoantes Corretas. Resultados: a análise mostrou maior concordância entre os grupos de juízas para os extremos das possibilidades de julgamento (leve e grave), sendo mais difícil o julgamento e classificação da gravidade levemente-moderada e moderadamente-grave. Conclusão: observou-se maior facilidade dos juízes na identificação e julgamento dos sujeitos com gravidade grave (maior) e leve (menor) na fala. As leigas possuem maior dificuldade para julgar com precisão a gravidade do desvio fonológico do que as mães e fonoaudiólogas.
\end{abstract}

DESCRITORES: Distúrbios da Fala; Criança; Julgamento

\section{INTRODUÇÃO}

A fala das crianças se desenvolve normalmente até os 5-6 anos quando não ocorrem alterações nos quesitos biológicos, afetivos e ambientais. Alterações no nível fonológico podem levar aos

(1) Fonoaudióloga; Bolsista do Conselho Nacional de Desenvolvimento Científico e Tecnológico, CNPq; Doutoranda em Linguística na Pontifícia Universidade Católica do Rio Grande do Sul, PUCRS, Porto Alegre, RS, Brasil.

(2) Fonoaudióloga; Professora do Curso de Graduação em Fonoaudiologia da Universidade Federal de Santa Maria, UFSM, Santa Maria, RS, Brasil; Mestre em Distúrbios da Comunicação Humana pela Universidade Federal de Santa Maria.

(3) Fonoaudióloga; Professora do Curso de Fonoaudiologia e do Mestrado em Distúrbios da Comunicação Humana da Universidade Federal de Santa Maria, UFSM, Santa Maria, RS, Brasil; Doutora em Linguística Aplicada pela Pontifícia Universidade Católica do Rio Grande do Sul.

(4) Fonoaudióloga; Professora do Curso de Fonoaudiologia e do Mestrado em Distúrbios da Comunicação Humana da Universidade Federal de Santa Maria, UFSM, Santa Maria, RS, Brasil; Doutora em Linguística Aplicada pela Pontifícia Universidade Católica do Rio Grande do Sul.

Conflito de interesses: inexistente desvios fonológicos, os quais podem ser classificados quanto à gravidade 1 .

A gravidade é a mensuração do quanto a capacidade de comunicação é inferior às expectativas, isto é, do quão está alterada a fala da criança em relação ao padrão adulto. De acordo com estudos ${ }^{2,3}$ a gravidade tem sido um dos aspectos clínicos mais negligenciados da fonologia, uma vez que muitos estudos não consideram os graus de gravidade no momento da intervenção, por exemplo, o que pode acarretar num planejamento terapêutico inadequado.

Uma pesquisa ${ }^{3}$ verificou os procedimentos utilizados por 333 fonoaudiólogos, para a avaliação da fala de crianças. A partir de um questionário foram verificadas questões quanto à formação, experiência, condições de trabalho dos fonoaudiólogos e, ainda, referentes aos critérios de avaliação e análise do sistema de sons de crianças, como por exemplo, o uso de testes comercializados, procedimentos de análise, tempo de avaliação, dentre outras. Com base nos resultados, os autores observaram que há necessidade de se encontrar métodos mais 
eficientes para avaliar e analisar a fala das crianças minuciosamente, pois a maioria dos fonoaudiólogos pesquisados despende muito tempo para avaliação.

Embora existam diferentes formas para avaliar o grau do desvio fonológico o mais utilizado em pesquisas atualmente ${ }^{4-12}$ é o percentual de consoantes corretas (PCC) ${ }^{1}$ a partir do qual o desvio pode ser classificado como grave, moderadamente-grave, levemente-moderado e leve.

Estudos têm sido realizados a fim de verificar a percepção do desvio fonológico pelos professores ${ }^{13} \mathrm{e}$ até mesmo a percepção da gravidade por fonoaudiólogos ${ }^{6}$, porém não há pesquisas que consideram o julgamento perceptivo da gravidade por diferentes grupos de julgadores (mães, leigas e fonoaudiólogas). Tal estudo torna-se importante para o entendimento do quanto as pessoas compreendem a fala de crianças com desvio fonológico, o que auxiliará no planejamento terapêutico do fonoaudiólogo e, principalmente, nas orientações preventivas tanto para a comunidade em geral (leigos) quanto durante o tratamento (mães).

O objetivo desta pesquisa foi comparar a gravidade do desvio fonológico a partir da análise perceptiva de três grupos distintos de julgadoras.

\section{MÉTODOS}

A pesquisa foi composta por duas amostras, uma julgada e outra julgadora. A amostra julgada foi constituída por 30 crianças com diagnóstico de desvio fonológico, na faixa etária de $4: 1$ a $7: 11$, sendo 20 do sexo masculino e 10 do sexo feminino.

A amostra julgadora, toda do sexo feminino, foi composta por cinco sujeitos sem contato diário com crianças com ou sem alterações de fala, no caso do desvio fonológico, e sem conhecimento sobre o assunto, grupo denominado de leigas; cinco fonoaudiólogas ou estudantes do último ano do Curso de Fonoaudiologia, grupo chamado de fonoaudiólogas; e cinco mães dos sujeitos julgados, grupo denominado de mães, formaram os grupos de juízes. Todas as adultas eram falantes nativas do Português Brasileiro (PB).

Os sujeitos julgados foram submetidos a avaliações fonoaudiológicas (avaliação da linguagem compreensiva e expressiva, avaliação do sistema estomatognático, exame articulatório, avaliação fonológica, constituída por coleta e posterior análise dos dados da fala, e audiológica).

A avaliação fonológica foi realizada utilizando as figuras do fichário do Teste ABFW ${ }^{14}$, o qual permitiu, por meio da nomeação e fala espontânea, a obtenção da amostra linguística da criança. Os dados foram gravados, transcritos foneticamente $e$ analisados.
A partir da análise contrastiva, determinou-se o sistema fonológico da criança. Após, foi calculado o índice Percentual de Consoantes Corretas (PCC) ${ }^{1}$.

Após a realização do PCC e classificação da gravidade do desvio fonológico, constatou-se que 13 sujeitos apresentavam desvio fonológico leve, 10 levemente- moderado, quatro moderadamentegrave e três grave.

Ainda, foi aplicada, por meio de gravuras temáticas retiradas da "Nova Dimensão em Produção de Textos" ${ }^{15}$, uma prova narrativa obtendo-se uma amostra de fala espontânea que pôde ser analisada pelos grupos de julgadoras. Três diferentes sequências lógicas (palhaço, bola e pato), com três figuras cada, foram utilizadas para a coleta das narrativas. As histórias narradas pelas crianças foram gravadas, transcritas e sofreram recortes excluindo-se possíveis interferências da pesquisadora durante as narrações e padronizou-se o tempo de apresentação de todas as narrativas para em torno de 20 segundos.

Após a edição das narrativas espontâneas das 30 crianças, as 90 narrativas (três narrativas de cada criança) foram gravadas em ordem aleatória em mídia digital (CD), com um intervalo de 10 segundos entre cada narrativa para posterior apresentação às julgadoras.

As 90 narrativas, gravadas no $C D$ na sequência da Fala 1 a Fala 90, foram apresentadas às julgadoras acompanhadas de um questionário com perguntas de identificação e caracterização das participantes, além da grade para marcação da gravidade. As possíveis marcações foram baseadas em um estudo ${ }^{6}$ as quais eram: leve (quando as alterações de fala dificultavam pouco o entendimento do que a criança dizia), levemente-moderado (quando as alterações de fala dificultavam em parte o entendimento do que a criança dizia), moderadamente-grave (quando as alterações de fala dificultavam muito o entendimento do que a criança dizia), grave (quando as alterações de fala não permitiam o entendimento do que a criança dizia).

Este estudo foi desenvolvido por meio do projeto registrado e aprovado no Comitê de Ética em Pesquisa (CEP) da Universidade Federal de Santa Maria sob $n^{\circ} 106 / 05$. A coleta dos dados teve início após leitura e assinatura do Termo de Consentimento Livre e Esclarecido pelos responsáveis das crianças e pelas participantes maiores de 18 anos.

A partir do julgamento dos grupos de juízas, pôde-se realizar a Moda (Mo) das 90 narrativas e estabelecer a Moda dos julgamentos para cada sujeito. No total, obteve-se a Moda das três narrativas dos 30 sujeitos julgados. Houve momentos em que não foi possível se ter uma Moda (Amodal) nos 
julgamentos entre as julgadoras ou para cada uma das narrativas ou, ainda, para cada sujeito.

A fim de verificar a associação entre as variáveis foi utilizada a Estatística Kappa e para verificar o grau de concordância foi utilizada a classificação ${ }^{16}$ : sem concordância $(<0.00)$; pobre $(0.00-0.19)$; regular $(0.20-0.39)$, moderado $(0.40-0.59)$; substancial $(0.60-0.79)$; quase perfeito $(0.80-1.00)$.

\section{RESULTADOS}

$\mathrm{Na}$ Tabela 1 são apresentados os resultados referentes à gravidade julgadas e a concordância dos grupos de fonoaudiólogas, mães e leigas.

Pode-se observar que o grau de concordância entre o grupo de fonoaudiólogas para as gravidades leve e grave foi moderado. Esse grupo teve pobre concordância para a gravidade levemente-grave e moderadamente-grave. Para os sujeitos sem Moda, não houve concordância $(-0,053)$.

No grupo de mães verifica-se concordância regular para as gravidades leve e grave e pobre para a gravidade moderadamente-grave e para os sujeitos sem Moda $(0,0050)$. Não houve concordância para a gravidade levemente-moderada.

Para o grupo de leigas a concordância foi moderada nos graus grave e leve. A concordância entre esse grupo foi regular para a gravidade moderadamente-grave e pobre para a gravidade levemente- moderada. Não houve concordância para os sujeitos sem Moda $(-0,032)$.

Na Tabela 2 são apresentadas as concordâncias entre os grupos de julgadoras (fonoaudiólogas $x$ mães, fonoaudiólogas $x$ leigas e mães $x$ leigas) para a gravidade.

Entre os grupos de fonoaudiólogas e mães, observa-se uma concordância quase perfeita no julgamento do grau leve. O grau de concordância entre esses grupos foi substancial para a gravidade grave, e foi regular para a gravidade moderadamente-grave e para os sujeitos sem Moda $(0,265)$. Para o julgamento da gravidade levemente-moderada, não houve concordância entre fonoaudiólogas e mães.

Os grupos de fonoaudiólogas e leigas tiveram concordância substancial para o julgamento dos graus leve e grave. O grau de concordância entre esses grupos foi pobre para a gravidade levemente-moderada e para os sujeitos sem Moda $(0,148)$. Não houve concordância entre as fonoaudiólogas e leigas para a gravidade moderadamente-grave.

Os grupos de mães e leigas tiveram concordância moderada no julgamento das gravidades leve e grave. Para as gravidades levemente-moderada e moderadamente-grave, a concordância foi pobre. $\mathrm{E}$ para os sujeitos sem Moda foi regular $(0,265)$.

Tabela 1 - Gravidade dos sujeitos estudados e a concordância, segundo o julgamento dos grupos de julgadoras

\begin{tabular}{cccccccccc}
\hline Gravidade & \multicolumn{1}{c}{ Fonoaudiólogas } & \multicolumn{3}{c}{ Mães } & \multicolumn{3}{c}{ Leigas } \\
\cline { 2 - 9 } & $\%$ & $\mathbf{n}$ & $\mathbf{c}$ & $\%$ & $\mathbf{n}$ & $\mathbf{c}$ & $\%$ & $\mathbf{n}$ & $\mathbf{c}$ \\
\hline $\begin{array}{c}\text { Grave } \\
\begin{array}{c}\text { Moderadamente- } \\
\text { grave }\end{array}\end{array}$ & 6,7 & 2 & 0,502 & 10,0 & 3 & 0,206 & 13,3 & 4 & 0,526 \\
$\begin{array}{c}\text { Levemente- } \\
\text { moderado } \\
\text { Leve }\end{array}$ & 20,0 & 6 & 0,159 & 10,0 & 3 & 0,029 & 30,0 & 9 & 0,256 \\
& 26,7 & 8 & 0,055 & 13,3 & 4 & $-0,078$ & 13,3 & 4 & 0,058 \\
\hline
\end{tabular}

Legenda: c: Concordância

Tabela 2 - Grau de concordância da gravidade entre os grupos de julgadoras

\begin{tabular}{cccc}
\hline Gravidade & $\begin{array}{c}\text { Fonoaudiólogas } \mathbf{x} \\
\text { Mães }\end{array}$ & $\begin{array}{c}\text { Fonoaudiólogas } \mathbf{x} \\
\text { Leigas }\end{array}$ & Mães x Leigas \\
\hline Grave & 0,783 & 0,634 & 0,516 \\
Moderadamente-grave & 0,359 & $-0,14$ & 0,02 \\
Levemente-moderado & $-0,19$ & 0,048 & 0,135 \\
Leve & 0,83 & 0,71 & 0,516 \\
\hline
\end{tabular}


Tabela 3 - Grau de concordância geral entre as julgadoras

\begin{tabular}{cc}
\hline Gravidade & Julgadoras \\
\hline Grave & 0,63 \\
Moderadamente-grave & 0,028 \\
Levemente-moderado & $-0,015$ \\
Leve & 0,689 \\
\hline
\end{tabular}

$\mathrm{Na}$ Tabela 3 está apresentado o grau de concordância da gravidade entre todas as julgadoras (fonoaudiólogas $\mathrm{x}$ mães $\mathrm{x}$ leigas).

O grau de concordância entre todos os grupos de julgadoras foi substancial para as gravidades leve e grave. Ainda, observa-se que a gravidade moderadamente-grave teve grau de concordância pobre, enquanto que não houve concordância para gravidade levemente-moderada.

\section{DISCUSSÃO}

Observou-se que, no grupo de fonoaudiólogas (Tabela 1), o conceito de gravidade leve foi julgado para oito sujeitos. Oito crianças julgadas não tiveram Moda para suas narrativas. Constatou-se certa dificuldade no julgamento da gravidade do desvio fonológico por este grupo, já que um número considerável de crianças não teve suas modas calculadas.

Em um estudo ${ }^{6}$ realizado com fonoaudiólogos e estudantes de fonoaudiologia, foi observada que a maior parte dos sujeitos foi classificada como leve e levemente-moderado, o que vai ao encontro dos achados desta pesquisa para a gravidade do desvio fonológico.

O grau de concordância entre o grupo de fonoaudiólogas, para as gravidades leve e grave foi moderado. Esse grupo teve pobre concordância para a gravidade moderadamente-grave e levemente-moderada, concordando com os achados de uma pesquisa ${ }^{6}$ em que as autoras obtiveram 0 mesmo resultado para estas duas gravidades. Para os sujeitos sem Moda, não houve concordância $(-0,053)$ da gravidade do desvio fonológico.

De acordo com uma pesquisa ${ }^{2}$ os fonoaudiólogos mais experientes, parecem prestar atenção ao número, tipos de erros e à inteligibilidade de fala para classificar a gravidade do desvio, também observam o nível da palavra e os segmentos ausentes.

No grupo de mães, oito crianças foram classificadas com grau leve. Não houve Moda de 12 sujeitos julgados por este grupo. Tais achados se justificam pelo fato de que as mães possuem experiência com a fala desviante, o que torna o padrão familiar quando o ouvinte se acostuma com ele ${ }^{17,18}$.

Os pais têm um envolvimento considerável durante o processo avaliativo, sendo isto essencial na conscientização da importância do tratamento ${ }^{3}$. O que se percebe na clínica fonoaudiológica é que muitas vezes os pais estão tão habituados com a fala "errada" da criança que deixam de procurar o atendimento, sendo mais tarde encaminhados por terceiros.

O grupo de leigas classificou nove crianças com grau moderadamente-grave, cinco com grau leve, quatro com grau levemente-moderado, e quatro com grau grave. Para oito sujeitos não houve Moda das suas narrativas. A concordância entre o grupo foi moderada nos graus grave e leve; regular para a gravidade moderadamente-grave e; pobre para a gravidade levemente-moderada. Para os sujeitos sem Moda, o grupo não teve grau de concordância $(-0,032)$.

De acordo com um estudo ${ }^{18}$, crianças com desenvolvimento de fala normal com média de idade de quatro anos, são $93 \%$ inteligíveis para ouvintes leigos.

Os grupos de fonoaudiólogas e mães julgaram de maneira semelhante a gravidade do desvio fonológico das crianças participantes da pesquisa (Tabela 2), sendo que a maior concordância foi para o grau leve, o qual seria mais tolerável para ouvintes com experiência com falas desviantes ${ }^{17}$. Provavelmente, o fato da concordância no julgamento da gravidade dos desvios fonológicos por esses grupos ter sido quase perfeita ou substancial deve-se à facilidade em observar os "erros" cometidos pela criança em sua fala. Características do ouvinte como, familiaridade com o falante e familiaridade com crianças com desvio fonológico ${ }^{18,19}$ influenciaram tais resultados.

Nos grupos de fonoaudiólogas e leigas observou-se maior concordância e, consequentemente a maior facilidade, nos julgamentos e identificações das gravidades leve e grave, demonstrando que tanto para sujeitos com experiência em classificações de falas desviantes (fonoaudiólogas) quanto os sujeitos sem essa experiência (leigas) há um julgamento mais fácil dos extremos. Esse achado discorda parcialmente dos achados de uma pesquisa ${ }^{6}$ em que as autoras verificaram que os graus leve e levemente-moderado foram mais utilizados pelas julgadoras.

Nos grupos de mães e leigas verificou-se maior concordância, e até mesmo mais facilidade, no julgamento das possibilidades dos extremos (leve e grave). Mesmo um grupo tendo maior contato com crianças com fala desviante (mães) e outro não (leigas). 
Através da observação da Tabela 3, que a concordância entre os grupos de fonoaudiólogas, mães e leigas foi mais acentuada (substancial) para os julgamentos da gravidade leve e grave, demonstrando ser mais facilmente julgadas em suas extremidades. Supõe-se que este fato deve-se à fragilidade da distinção perceptual entre as gravidades do desvio fonológico.

As gravidades leve e grave são mais facilmente julgadas, sendo mais difícil o julgamento e classificação do desvio fonológico levemente-moderado e moderadamente-grave. $\mathrm{O}$ grupo de fonoaudiólogas e mães utilizou mais o grau leve em seus julgamentos, enquanto que o grupo das leigas fez maior uso do grau levemente-grave. A concordância entre os grupos foi mais acentuada nos extremos (leve e grave) de sua classificação. As leigas possuem maior dificuldade para julgar com precisão a gravidade do desvio fonológico do que as mães e fonoaudiólogas.

\section{CONCLUSÃO}

Os achados desta pesquisa permitem concluir que, pelo fato de o fonoaudiólogo apresentar experiência clínica com crianças com desvio fonológico, ele é capaz de compreender mais facilmente as alterações de fala, já as mães percebem de forma semelhante ao fonoaudiólogo a dificuldade de fala, porém esta percepção está relacionada ao convívio diário com o filho. Em compensação, as leigas apresentam algumas limitações, justamente por não manterem nenhum contato. Verifica-se, também, a dificuldade dos grupos para julgar as gravidades levemente-moderada e moderadamente-grave, justamente por estarem bastante próximas aos extremos, leve e grave, necessitando, desta maneira, de análises mais minuciosas.

\begin{abstract}
Purpose: to compare the severity of phonological disorder from the perceptual analysis of three distinct groups of judges. Methods: the research consisted of two samples, one to be assessed with 30 individuals with phonological disorder with ages between 4:1 to 7:11, and another sample that comprises the judges (five speech therapists, five laypeople and five mothers). We evaluated the spontaneous speech of the children through a three logical sequence narrative to be assessed by the judges, accompanied by a grid where to register the severity level. For data analysis, the mode of 90 pieces of narratives was carried through, making possible the statistical analysis of the data based on Kappa Concordance Analysis. Results: the analysis showed higher concordance level among the groups of judges in the extremes of the possibilities of assessment (mild and severe), being more difficult the judgment of mild-moderate and moderate-severe. Conclusion: we observed that it was easier for judges to act in the identification and judgment of those subjects with severe (higher) and mild (lower) speech severities. The laypeople have more difficulty to judge accurately the severity of phonological disorder than mothers and speech therapists.
\end{abstract}

KEYWORDS: Speech Disorders; Child; Judgment

\section{REFERÊNCIAS}

1. Shriberg LD, Kwiatkowski J. Phonological disorders I: a diagnostic classification system. J Speech Hear Dis. 1982a; 47:226-41.

2. Flipsen Junior $P$, Hammer JB, Yost KM. Measuring severity of involvement in speech delay: segmental and whole-word measures. Am J Speech Lang Path. 2005; 14(4):298-312.

3. Skahan SM, Watson M, Lof GL. Speech-language pathologists' assessment practices for children with suspected speech sound disorders: results of a national survey. Am J Speech Lang Path. 2007; 16:246-59.
4. Wertzner HF, Ramos ACO, Amaro L. Índices fonológicos aplicados ao desenvolvimento fonológico típico e ao transtorno fonológico. Rev Soc Bras Fonoaudiol. 2004; 9(4):199-204.

5. Gillon GT. Facilitating phoneme awareness development in 3- and 4-year-old children with speech impairment. Lang Speech Hear Serv Sch. 2005; 36(4):308-24.

6. Wertzner HF, Amaro L, Teramoto SS. Gravidade do distúrbio fonológico: julgamento perceptivo e porcentagem de consoantes corretas. Pró-Fono. 2005; 17(2):185-94.

7. Wertzner HF, Amaro L, Galea DES. Phonological performance measured by speech severity indices 
compared with correlated factors. SP Med J. 2007; 125(6):309-14.

8. Mota HB, Keske-Soares M, Bagetti T, Ceron MI, Melo Filha MGC. Análise comparativa da eficiência de três diferentes modelos de terapia fonológica. Pró-Fono. 2007; 19(1):67-74.

9. Keske-Soares M, Brancalioni AR, Marini C, Pagliarin KC, Ceron MI. Eficácia da terapia para desvios fonológicos com diferentes modelos terapêuticos. Pró-Fono. 2008; 20(3):153-8.

10. Wertzner HF, Alves RR, Ramos ACO. Análise do desenvolvimento das habilidades diadococinéticas orais em crianças normais e com transtorno fonológico. Rev Soc Bras Fonoaudiol. 2008; 13(2):136-42.

11. Barberena LS, Keske-Soares M, Mota HB. Generalização baseada nas relações implicacionais obtida pelo modelo "ABAB-Retirada e Provas Múltiplas". Rev Soc Bras Fonoaudiol. 2008; 13(2):143-53.

12. McIntosh B, Dodd B. Evaluation of core vocabulary intervention for treatment of inconsistent phonological disorder: three treatment case studies. Child Lang Teach Ther. 2008; 24(3):307-27.
13. Overby M, Carrel T, Bernthal J. Teachers' perceptions of students with speech sound disorders: a quantitative and qualitative analysis. Lang Speech Hear Serv Sch. 2007; 38(4):327-41. 14. Andrade C, Befi-Lopes D, Fernandes F, Wertzner $H$. ABFW: Teste de linguagem infantil nas áreas de fonologia, vocabulário, fluência e pragmática. São Paulo: Pró-Fono; 2000.

15. Almeida Z. Nova dimensão em produção de textos. Belo Horizonte: Dimensão; 1993.

16. Landis JR, Koch GG. The measurement of observer agreement for categorical data. Biometrics. 1977; 33(1):159-74.

17. Shriberg LD, Kwiatkowski J. Phonological disorders III: a procedure for assessing severity of involvement. J Speech Hear Disord. 1982; 47(3):256-70.

18. Gordon-Brannan M. Assessing intelligibility: children's expressive phonologies. Top Lang Disord. 1994; 14(2):17-25.

19. Yavas MS, Lamprecht RR. Os processos e a inteligibilidade na fonologia com desvios. In: Yavas MS. Desvios Fonológicos em crianças: teoria, pesquisa e tratamento. Porto Alegre: Mercado Aberto; 1990. 264p.

RECEBIDO EM: 04/02/2009

ACEITO EM: 03/08/2009

Endereço para correspondência:

Karina Carlesso Pagliarin

Rua Coronel Scherer, 09

São Pedro do Sul - RS

CEP: 97400-000

E-mail: karinap_fono@yahoo.com.br 